\title{
La Révolution batave : un cas particulier dans la grande famille des républiques sœurs?
}

The Batavian Revolution : a specific case in the large family of Sister Republics?

Annie Jourdan

\section{(2) OpenEdition \\ 12 Journals}

Édition électronique

URL : https://journals.openedition.org/ahrf/13364

DOI : 10.4000/ahrf.13364

ISSN : 1952-403X

\section{Éditeur :}

Armand Colin, Société des études robespierristes

\section{Édition imprimée}

Date de publication : 1 décembre 2014

Pagination : 73-96

ISBN : 978-2-200-92928-2

ISSN : 0003-4436

\section{Référence électronique}

Annie Jourdan, « La Révolution batave : un cas particulier dans la grande famille des républiques sœurs? ", Annales historiques de la Révolution française [En ligne], 378 | octobre-décembre 2014, mis en ligne le 01 décembre 2014, consulté le 01 juillet 2021. URL : http://journals.openedition.org/ahrf/ 13364 ; DOI : https://doi.org/10.4000/ahrf.13364 


\title{
LA RÉVOLUTION BATAVE : UN CAS PARTICULIER DANS LA GRANDE FAMILLE DES RÉPUBLIQUES SEERS?
}

Annie JOURDAN

\begin{abstract}
Dans la « grande famille » des républiques sœurs, la République batave figurent en première place : par sa date, son républicanisme séculaire, et son autonomie relative. Elle aurait pu être un modèle pour celles qui suivent entre 1796 et 1799. II n'en a rien été. Trois ans et deux coups d'État pour introduire une constitution et un gouvernement stable, voilà qui était inquiétant ! Une comparaison entre la République batave et les républiques dites sœurs démontre combien le Directoire, peu interventionniste, voire hésitant en ses débuts, non seulement a tiré des leçons des années précédentes et freiné les velléités " terroristes », latentes dans les républiques nouvelles en leur évitant une guerre civile, mais fut en conséquence beaucoup plus présent en Suisse et en Italie, souvent, à la demande expresse des patriotes du lieu. Ces derniers jouent un rôle aussi important que les agents et les généraux français. Prendre en compte leurs intrigues permet de nuancer tout à la fois les interprétations courantes sur la politique extérieure du Directoire et celles sur les républiques sœurs.
\end{abstract}

Mots-clés : Directoire, république sœur, République batave, Genève, Italie, traités, constitutions.

«C'est une chose inimaginable que de voir un peuple impatient d'être conquis et prier pour le succès de ses ennemis ${ }^{1}$. John Quincy Adams, ambassadeur des États-Unis à La Haye, décrit en ces termes l'ambiance qui précède l'arrivée des Français en Hollande. En ce mois

(1) John Quincy ADAMS, The Writings of John Quincy Adams, 7 vols., New York, 1968, I, p. 226. Sera abrégé ADAMS. 
de novembre 1794, Adams n'est qu'au début de ses surprises. Durant les deux mois que dure la campagne de Hollande, il a amplement le temps de s'étonner et de constater que « l'arrivée des Français est attendue plus ardemment que le Messie par les enfants d'Abraham ». Les patriotes font tout leur possible pour faciliter l'arrivée des Français ${ }^{2}$. Récemment nommé en Hollande, Adams avait d'abord visité la Grande-Bretagne, et avait été choqué par le caractère inhumain des mesures mises en œuvre pour affamer la France révolutionnaire. Une fois à Amsterdam, il s'était aperçu que régnait là aussi l'intolérance vis-à-vis des dissidents politiques. L'époque décidément n'était pas à la concorde : des hommes avaient été emprisonnés, condamnés aux travaux forcés, et perpétuellement bannis pour avoir soumis une pétition. Heureusement pour les patriotes hollandais, les Français arrivèrent le 17 janvier à Utrecht ; le 19, ils étaient à Amsterdam. Nouvel étonnement du jeune Adams : "Une révolution a eu lieu, dont le contenu était plus ou moins prévisible, mais dont les formes ont été infiniment plus douces que tout ce que l'on pouvait imaginer $»^{3}$. Tout cela surprend donc le diplomate américain. On lui avait parlé de Français fanatiques, sanguinaires et barbares, Adams assiste à un déploiement de forces tout en douceur. Les conditions même de cette libération et son paisible déroulement constituent une première dans l'histoire occidentale. La Révolution batave s'affiche d'emblée comme spécifique. Les observations d'Adams le confirment, d'autant que son engouement pour la République française n'est pas inconditionnel.

\section{Comment en est-on arrivé là ?}

La France n' avait pourtant pas prévu de révolutionner l'Europe. Dans son décret du 22 mai 1790, elle proclamait au contraire ne plus vouloir faire de conquêtes et déclarait la paix au monde. Quelques mois plus tard, il est vrai, un glissement se faisait jour - le 28 octobre 1790 : sur le droit des peuples à réclamer leur pleine souverainetét. Le décret du 19 novembre 1792 poursuivait en ce sens : la Convention déclarait son intention d'assister les peuples qui réclameraient leur liberté - au grand dam de l'Angleterre ${ }^{5}$.

(2) Ibidem, I, p. 241-242.

(3) Ibid., I, p. 234 et p. 276.

(4) Eddy Kolla, Legality, Legitimacy, and the Will of the People: the French Revolution and the Transformation of International Law. 1789-1792, PhD-thesis John Hopkins University, 2010, 174-181 ; Marc BELISSA, Fraternité universelle et Intérêt national. Les cosmopolitiques du droit des gens, Paris, Kimé, 1998, p. 220-223 ; Hervé LEUWERS, Un juriste en politique. Merlin de Douai (1754-1838), Arras, Artois Presses Université, 1996, p. 218-222.

(5) William PITT jr., TheWar Speeches, Oxford, Clarendon Press, 1949, p. 24-51. 
Dès 1789, cependant, la Corse avait été réunie à la France, sans qu'il lui soit demandé son avis. Ce genre d'initiatives n'était pas inédit. L'Ancien Régime faisait souvent fi du droit des gens. En 1772, la Pologne avait ainsi été partagée entre les trois grandes puissances de l'est de l'Europe ; en 1763, la Prusse avait définitivement annexé la Silésie qui appartenait à l'Autriche. Mais la Révolution française avait semblé annoncer autre chose : le respect du droit des peuples à leur souveraineté. Ce fut pour peu de temps. D'une réunion à l'autre, de 1790 à 1793, la République française prit l'habitude d'arrondir ses frontières et de désenclaver son territoire ${ }^{6}$, sous prétexte que les peuples impliqués souhaitaient devenir Français afin d'accéder à la liberté. En automne 1792, ce fut le tour de la Savoie. L'incident avait provoqué une révolution dans la république de Genève qui était limitrophe et qui avait craint un moment d'être elle-même « réunie ». La Révolution genevoise en découle ${ }^{7}$. Lors de la première campagne de Belgique de l'hiver 1792-1793, les peuples liégeois et belge demandèrent la réunion à la France; des assemblées primaires furent organisées qui semblaient opiner dans ce sens ${ }^{8}$. Dès lors, le Brabant néerlandais connut une première « libération », avec ses arbres de la Liberté et ses drapeaux tricolores. La libération fut de courte durée, tout comme l'incorporation de la Belgique à la France ${ }^{9}$. En mars 1793, Dumouriez était battu à Neerwinden. Les patriotes des Pays-Bas autrichiens et des Provinces-Unies retournaient dans le giron de leurs maîtres et « despotes ». La première tentative d'émancipation des peuples européens se concluait par un échec.

Peut-être cet échec explique-t-il pourquoi la Convention mit un frein à la politique de « libération ». Le 13 avril 1793, elle affirmait ne plus vouloir s'immiscer en aucune façon dans le gouvernement des puissances étrangères ${ }^{10}$. Le 17 novembre suivant, sur un rapport de Robespierre

(6) Les enclaves allemandes de l'Alsace, de même que le Comtat Venaissin et Avignon. Sur l'incompatibilité entre souveraineté du peuple et droit international, et le refus de l'Europe d'accepter ces nouveautés. Voir note précédente et Eddy KoLlA, « Legality, Legitimacy, and the Will of the People », op.cit., p. 277-284.

(7) Par défaut d'espace, nous n'aborderons pas le problème de la Rhénanie. Jean-Louis HAROUEL, Les Républiques sæurs, Paris, PUF, 1997, p. 30-34. Éric GOLAY, Quand le peuple devint roi. Mouvement populaire, politique et révolution à Genève, Genève, Slatkine, 2001. Et Raymond GuYOT, Le Directoire et la paix de l'Europe, des traités de Bâle à la deuxième coalition, Paris, Félix Alcan, 1911.

(8) Marc BeLISSA, Fraternité universelle, op. cit., p. 314-360 ; Patricia Chastain HowE, Foreign Policy and the French Revolution. Charles-François Dumouriez, Pierre Lebrun and the Belgian Plan, 1789-1793, New York, Palgrave, 2008, p. 111-114.

(9) Joost RosendaAl et Anton VAN DE SANDE (dir.) Dansen rond de vrijheidsboom. Revolutionaire cultuur in Brabant en de Franse invasie van 1793, Bois-le-Duc, 1993.

(10) Voir Virginie MARTIN, «La République a-t-elle voulu la guerre? », L'Histoire, n 60, 2013, p.76-80 ; Marc BELISSA, Fraternité universelle, op. cit., p. 371-377. 
concernant les relations de la République française avec les nations étrangères, la France renonçait à la guerre de propagande et de conquête qui avait caractérisé la période dite girondine ${ }^{11}$ - et annonçait sa volonté de respecter le territoire des nations alliées et neutres ${ }^{12}$. Ainsi était freinée pour un bref laps de temps la politique d'émancipation des peuples opprimés ${ }^{13}$. Elle reprit au cours de l'année suivante, mais les législateurs français persistèrent à l'envisager comme une libération souhaitée par les peuples, et non comme une politique de conquête.

La France ne fut pas toujours à l'origine des projets de réunion ou de libération. Un deuxième point fondamental dans ce contexte est le rôle essentiel qu'ont joué les patriotes étrangers, réfugiés en France. Parmi eux, les Bataves, qui depuis leur exil de 1787, ont tout fait pour provoquer une intervention dans leur pays. Ce sont eux qui les premiers ont conçu des projets pour révolutionner leur patrie avec l'aide des Français ${ }^{14}$. L'entrée en guerre d'avril 1792 est en partie due aux incitations des patriotes réfugiés en France, de quelque nationalité qu'ils soient ${ }^{15}$. Pour l'autre, elle a été stimulée par le chef girondin, Brissot, chez qui s'entremêlaient messianisme révolutionnaire et stratégie politique. Brissot espérait que la guerre oblige Louis XVI à dévoiler ses véritables sentiments, tout en libérant les peuples " opprimés ». Les intrigues récurrentes des étrangers ont influé sur l'opinion des Français et n'ont pu que les conforter dans ce messianisme. Dans ce cas précis, ils étaient persuadés d'être appelés par les Hollandais. Et ils le furent effectivement au cours de l'été 1794. Ils ne faisaient donc pas la guerre à une population, mais à un gouvernement ${ }^{16}$.

(11) Virginie MARTIN minimise l'aspect propagandiste et lit dans cette politique une volonté de défense nationale, avant d'insister sur les responsabilités des généraux. Nous y ajoutons celles des exilés ou patriotes étrangers. « Diplomatie et République : gageure ou impasse ? », dans Michel BIARD et alii (dir.), 1792. Entrer en République, Paris, Armand Colin, 2013, p. 283-296.

(12) Maximilien RobeSPIERRE, Euvres de Maximilien Robespierre, 10 vols., Paris, PUF, 1967, X, p.183-184 ; Marc BeLISSA, Fraternité universelle, op. cit., p. 389-392.

(13) Le 15 septembre 1793, la Convention décrète du reste qu'elle renonce à toute idée philanthropique et qu'elle exercera les lois ordinaires de la guerre. Archives Parlementaires, $1^{\mathrm{e}}$ série, 1789-1799, Laurent, J. Madival et E. LaURent (eds), Paris, Dupont-CNRS depuis 1867, vol. 74, p. 231 .

(14) Annie Jourdan, « Les Gaulois en Batavie : des relations diplomatiques machiavéliques » dans Annie JOURDAN et Joep LEERSSEN (dir.), Remous révolutionnaires, République batave, armée française, Amsterdam, AUP, 1996, p. 91-117.

(15) Orient LEE, Les comités et le club des patriotes belges et liégeois (1791-an III, Paris, 1931. Arianne MÉAUTIS, Le club helvétique de Paris (1790-1791) et la diffusion des idées révolutionnaires en Suisse, Neuchatel, 1969 ; Joost RosendaAL, Bataven. De Nederlandse vluchtelingen in Frankrijk, Nijmegen, 2005 ; Anna Maria RAO, Esuli. L'emigrazionepoliticaitaliana in Francia (1792-1802), Naples, 1992.

(16) Voir Marc BELISSA, «Faire la guerre au stadhouder ou à la nation hollandaise ? : le débat politique autour de la déclaration de guerre » dans Annie JOURDAN et Joep LEERSSEN, Remous révolutionnaires, p. 69-80. 
Une autre spécificité de la révolution batave réside dans le fait qu'elle a été préparée par les patriotes demeurés sur place, durant tout l'été et l'automne $1794^{17}$. Les résultats démontrent qu'ils ont bien fait leur travail et facilité l'avancée des troupes franco-bataves. Ce qui a d'emblée limité les violences. Ainsi que le constate Adams, 70000 soldats sur le pied de guerre et aucune violence, aucun sang versé ${ }^{18}$, cela tenait du miracle! En quelques jours, les municipalités étaient « régénérées », quelques semaines plus tard, ce furent les provinces. Des comités de sûreté et de surveillance étaient créés. Tout cela dans le plus grand calme - du moins pour un temps.

Enfin, l'époque était propice à la modération dans la victoire. Le régime qu'il est convenu d'appeler la Terreur venait d'être renversé. Durant les mois qui suivent, les Français rêvent de pacification et de modération, d'ordre et de prospérité. Les sociétés populaires sont muselées, et l'extrémisme freiné. Les journées populaires de germinal et prairial an III, qui allaient raviver les tendances répressives n'ont pas encore eu lieu elles se déroulent au moment même où Sieyès et Reubell concluent à La Haye le traité de paix. Et surtout, à cette date, les militaires sont encore sous la domination des civils. Ils sont contrôlés par des commissaires, eux-mêmes plutôt honnêtes et zélés, qui, le $1^{\text {er }}$ pluviôse an III, proclament l'indépendance du peuple batave et sa liberté à se donner le gouvernement qu'il lui plaira ${ }^{19}$.

Le contexte est primordial. L'an III n'est pas l'an II, mais pas non plus l'an IV, quand, sur l'exemple de Bonaparte, les militaires s'emparent progressivement de la souveraineté, et qu'à leurs côtés, agents, commissaires et fournisseurs spolient les pays libérés - notamment la Suisse, et avant tout l'Italie - «le dogme », soupirent alors les bons patriotes, est de $«$ faire sa fortune dans six mois $»^{20}$. Les Provinces-Unies ont donc la chance d'être « libérées » dans un contexte très favorable.

Un dernier point non moins important est qu'il s'agit de la première « république sœur ». Jusque-là, la République française avait avant tout

(17) Theodorus JoRISSEN, De Patriotten te Amsterdam in 1794, Amsterdam, 1875.

(18) Il y en a eu cependant durant la campagne de 1793-1794. Pieter GEYL, Geschiedenis van de Nederlandse stam, 6 vols., Amsterdam/Anvers, Wereldbibliotheek, 1958, vol. 6, p.1465-1517.

(19) Le Moniteur, 23, p. 393, Herman COLENBRANDER, Gedenkstukken der algemeene geschiedenis van Nederland van 1795 tot 1840, 10 vols., La Haye, 1905-1922, vol. I, p. 592. Abrégé $G S$, I.

(20) AN, AF III-185, Pièce 49 sur les dilapidations révoltantes qui s'opèrent en Italie, par le citoyen Reboul( lettre du 11 ventôse an IV). Voir aussi Jacques GODECHOT, Les commissaires aux armées sous le Directoire, 2 vols., Paris, 1937. 
annexé des territoires enclavés ou limitrophes ${ }^{21}$. C'était donc une nouveauté. Qui plus est, les Français trouvaient là une ancienne république, et ils en étaient conscients. Depuis deux siècles, et comme Mirabeau l'avait rappelé dans son livre Aux Bataves sur le Stadhouderat, les Hollandais avaient lutté pour conserver leur liberté. Ils étaient les seuls en Europe, avec la Suisse et Genève, à avoir su perpétuer un gouvernement républicain non aristocratique ${ }^{22}$ et à avoir connu des remous révolutionnaires avant même la prise de la Bastille. Certes, l'échec de la révolution patriote des années 1780 avait prouvé au monde que les Bataves modernes n'avaient plus l'énergie de leurs ancêtres, les Gueux du XVI ${ }^{\mathrm{e}}$ siècle. Néanmoins, la rapidité avec laquelle s'était effectuée la révolution de janvier 1795 aurait pu permettre à la République batave de devenir un modèle pour la future Europe républicaine. On verra plus loin ce qu'il en fut précisément.

\section{Carnot et les ambitions de la France}

La modération française vis-à-vis des Provinces-Unies ne fut pas accueillie unanimement à Paris. Plusieurs députés se plaignirent que la République française n'ait pas tiré d'une « si brillante expédition » tous les avantages qu'elle semblait promettre. Le 2 ventôse an III, alors que les bases du traité sont en train d'être formulées, Carnot rétorque aux critiques de ses collègues, en rappelant l'histoire récente des Provinces-Unies, où « la masse du peuple batave » aspirait depuis plus longtemps à une véritable alliance avec la France. Il remémore l'alliance de 1785 avec les patriotes, et l'abandon d'un gouvernement « lâche et fourbe »- entendez Louis XVI et Vergennes - qui a permis aux Prussiens de remettre la Hollande sous le joug. Il n'oublie pas non plus de mentionner la déclaration de guerre du $1^{\text {er }}$ février 1793 qui s'adressait au stadhouder, et non au peuple batave. Qui plus est, rappelle Carnot, les patriotes ont soutenu les troupes françaises. Grâce à eux, la campagne a été accélérée. Pour rassurer les sceptiques, Carnot conclut que le parti définitif ne sera pris qu'après la signature d'un « traité général, lequel établira les vrais rapports de la France avec les sept provinces ». Et de se féliciter des avantages à venir : subsistances, marine, forteresses, «tout est conservé [....] Nous avons acquis la navigation de trois grands fleuves, une marine importante est à notre disposition, des

(21) La Corse étant l'exception qui confirme la règle - d'autant que son « annexion » date de plus loin. S'y ajoutent à l'automne 1792 Nice et Monaco. Voir Jean-Louis HAROUEL, Les républiques sœurs, op.cit., p. 7, note 1. oligarchiques.

(22) Par rapport à la République de Venise ou à celle de Gênes qui passaient pour être 
ports, etc. ». Il est conscient cependant que, pour en profiter pleinement, « il ne faut pas abuser». Les avantages doivent être réciproques, afin qu'ils «ne soient pas éphémères ${ }^{23}$. C'est que les politiques français savent fort bien que la masse des richesses hollandaises « est transportable à volonté ». Toute mesure exorbitante ou injuste ferait fuir les capitaux à l'étranger. Carnot était réaliste. Sans doute avait-il suivi les discussions antérieures relatives à la spécificité des richesses néerlandaises ${ }^{24}$.

L'étape suivante est celle qui réunit Reubell et Sieyès à leurs homologues néerlandais de La Haye et qui conclut les négociations en faveur d'un traité de paix. De retour à Paris, Sieyès se plaît à en décrire les termes plus que favorables. La République française en effet a signé une alliance offensive et défensive, offrant « tous les avantages raisonnablement possibles [...] sans nuire à la bonne existence et à la dignité d'une nation devenue votre fidèle alliée ». Il se réjouit de la nouvelle puissance militaire et navale que confère à la France l'alliance. Bref, la République accède par là à « une grande et superbe existence navale et commerciale $»^{25}$. L'Assemblée nationale applaudit. Chacun pourtant avait mis de l'eau dans son vin. Parmi les exigences françaises, les négociateurs ont abandonné l'idée de placer la marine hollandaise sous commandement français et le prêt de 100 millions de florins. Certes, le prix à payer pour l'amitié française est lourd pour la petite république, mais il n'est pas insurmontable. John Quincy Adams, qui, au fil des mois devient de plus en plus critique vis-à-vis de la France, admet que la Hollande a été traitée « avec civilité et quelque générosité $»^{26}$.

L'alliance franco-batave promettait un renforcement des forces militaires et navales, propice à terminer rapidement la guerre avec l'Angleterre. Contrairement à la Belgique, qui allait être annexée à la République française, la Hollande, pour être utile à la France, devait rester indépendante. Dans une lettre au Comité de salut public du 7 brumaire an III, Portiez de l'Oise, qui connaissait bien la situation parce qu'il était sur place, le rappelait à son gouvernement. L'intérêt de la France était avant tout que les Hollandais soient rassurés et « qu'ils n'émigrent pas avec leurs trésors $»^{27}$. Portiez souligne enfin que les deux républiques sont moins rivales en vérité

(23) Le Moniteur, XXIII, p. 518-520.

(24) Des vrais intérêts de la France relativement à la Hollande, Paris, s.d. Voir aussi le Mémoire sur la Hollande, signé Ramel. GS, I, p. 612-617.

(25) Le Moniteur, XXIV, p. 516, p. 535-538, p. 618-619.

(26) ADAMs, I, p. 355. Rappelons que l'indemnité demandée par la France s'élevait tout de même à 100 millions de florins.

(27) AN, D XV-49, rapport du 7 brumaire an III. 
que complémentaires. D'une part, il y a « la Hollande, faible, économe et laborieuse »; d'autre part, « la France, forte, dissipatrice et consommante [sic !] ».Mais il déconseille de réunir la petite république à la grande ${ }^{28}$. Une réunion forcée nécessiterait de nombreuses armées et des dépenses considérables pour contraindre les « esprits et les cœurs » d'un peuple épris de liberté, et qui plus est, elle ferait dépasser à la France ses frontières naturelles. Alors qu'avec la Belgique, la réunion parait tout à fait logique, celle de la Hollande aurait été contre-nature.

Ces quelques remarques sur les motivations des uns et des autres démontrent avant tout que rien n'était déterminé d'avance, mais aussi que la France tenait à son alliance avec un pays qu' elle considérait complémentaire plus que rival. Elle souhaitait surtout qu'il lui soit contigu, de crainte qu'il ne soit une nouvelle fois menacé par la coalition et ne contribue pas à l'effort de guerre. Inversement, la Hollande était mécontente des conditions de l'alliance. Mais c'est surtout la crainte d'une détérioration croissante de leur patrie qui l'inquiétait. L'ouverture de l'Escaut notamment était vue comme un drame. Une fois le traité signé, les Bataves s'empressent toutefois d'organiser un gouvernement provisoire dans l'espoir de recouvrer au plus vite une stabilité politique et sociale. Or cela même s'avéra complexe.

\section{Une expérience inédite}

Au cours de son séjour en Hollande, le ministre de la France, François Noël, constatait que la situation n'était pas aussi idyllique qu'on ne l'avait pensé. Le contexte pour lui, c'est celui d'une république alliée, paralysée par les problèmes intérieurs. Aussi s'inquiète-t-il des troubles qui se succèdent dans les provinces et les villes, et qui retardent la remise de la contribution promise. En ce mois de novembre 1795, la France aurait dû en effet recevoir un acompte de 30 millions de florins qui se faisait attendre ${ }^{29}$. Noël tentait également de calmer les inquiétudes de la Frise et de la Zélande, qui ne voulaient pas d'une Assemblée nationale envahissant tous les pouvoirs. La zizanie sur ce point dura plus d'un an. Entre 1795 et 1797, les missives de Noël dépeignent à loisir les problèmes qui déchirent la république batave,

(28) Sur les rumeurs de réunion, voir Marc BELISSA, « Faire la guerre au stadhouder [...] ? », op. cit., p. 76-77. La réunion entre la Belgique et la Hollande était aussi envisagée. GS, II, 694-695 (Valckenaer).

(29) François CROUZET, « Aspects financiers de la relation franco-batave » dans Remous révolutionnaire, op.cit., p. 49-57. 
et insistent aussi sur l'incapacité où lui-même est d'agir, puisque, écrit-il en février 1797, « le gouvernement français dit ne pas vouloir intervenir $»^{30}$.

Durant trois ans, le ministre français est donc confronté aux querelles entre la Hollande et les autres provinces, aux menaces des ultrarévolutionnaires, aux révoltes populaires, aux mesures spoliatrices envisagées par les municipalités ${ }^{31}$, et au danger que la constitution batave ne soit pas unitaire. Auquel cas la république batave ne serait «d'aucun secours à la France ». Comme il est censé ne pas intervenir directement, Noël agit secrètement, notamment auprès de particuliers influents, tels que Bicker et Schimmelpenninck qu'il essaie de convertir à ses idées. La plupart du temps, il désespère des désaccords profonds qui déchirent les patriotes bataves et les mènent vers « une démagogie absurde ». Et d'avertir son gouvernement que si la République française abandonne la Hollande, elle tombera dans l'anarchie. Adams partage cet avis et décrit la situation de Hollande « comme ayant le même esprit de vengeance que partout ailleurs, le mépris des principes, la tendance à l'anarchie ». Les sociétés populaires surtout l'exaspèrent parce qu'elles tentent de dominer les autorités. Mais il s'irrite également des divisions partisanes qu'il découvre dans le plat pays et qui nuisent au patriotisme : «le sentiment national de ce pays est totalement subordonné à l'esprit de parti $»^{32}$.

Ce dont témoignent les agents français et les témoins étrangers, ce sont avant tout des oscillations et des lenteurs du gouvernement provisoire de Hollande ${ }^{33}$. Durant plus d'un an, l'Assemblée constituante a travaillé à un projet de constitution, présenté en août 1797 au peuple et rejeté par celuici. Dans un message de thermidor an V, Noël est perplexe à ce sujet. Que faut-il faire, puisque la France a reconnu l'indépendance de la Hollande ? Elle ne peut « violer l'usage que ses alliés font de leur liberté. Il faut donc encore une fois recourir au vœu du peuple ». En septembre 1797, tout est

(30) AN, AFIII-69. La discrétion en matière d'intervention est une stratégie récurrente de la France mais, en Hollande, l'intention était sincère. Le Directoire ne souhaitait pas imposer ses lois - si ce n'est l'unité et l'indivisibilité. Voir aussi Antonin DEBIDOUR, Recueil des actes du Directoire exécutif, 4 vols., Paris, 1910, II, p. 576-577.

(31) Les radicaux de Frise et d'Utrecht voulaient spolier les régents qui avaient soutenu la restauration orangiste de 1787. En Frise, soixante-dix personnes avaient ainsi été menacées de devoir payer 700000 florins, en tant qu'indemnités en faveur des patriotes fugitifs. Noël était intervenu, mais en vain. Quelques jours plus tard, il apprend que trente-sept d'entre elles ont été poursuivies et que trente vont devoir payer. AN AFIII-69.

(32) ADAMS, op.cit., I, p. 338.

(33) Voir la réaction de Wolfe TonE de décembre 1797, The Writings of Theobald Wolfe Tone, 3 vols., Oxford, Clarendon Press, 2007, III, p. 186-187, p. 193-194 ; Frédéric César de LA HARPE, Correspondance, 4 vols., Neufchatel, 1982-2004, I, 364 et 372. La Hollande est une leçon pour La Harpe, qui voudra éviter à tout prix la vacance du pouvoir. 
donc à refaire. Et tout laisse supposer de nouvelles lenteurs et de nouvelles querelles entre les factions. Noël s'inquiète de « la diversité et de la ténacité des opinions bataves bien plus grandes que chez aucun autre peuple ». Cet état de choses est suivi en France qui déplore d'avoir une alliée dépourvue de gouvernement. L'inquiétude s'accroît lors de la bataille de Kamperduin d'octobre 1797, où la marine hollandaise est battue par les Anglais ${ }^{34}$. Cette situation alarmante permet d'expliquer la promptitude avec laquelle Paris accepte le coup d'État du 22 janvier suivant. Le gouvernement batave faisait montre d'une inefficacité qui semblait tenir de la mauvaise volonté, voire de la trahison. Sans marine en état, la Hollande paraissait à la France une alliée de bien peu de valeur. Et d'autant plus qu'elle n'avait même pas de constitution et qu'elle était paralysée par des factions adverses. À l'instigation de Charles Delacroix qui succède à Noël, la France décide alors de soutenir le parti des radicaux ${ }^{35}$. Eux du moins font preuve d'énergie et dotent rapidement leur patrie d'une constitution démocratique.

Les Bataves avaient en vérité été devancés par la république de Genève, laquelle pourrait être qualifiée de première république sœur. Mais plus qu'une alliée indispensable de la France, Genève faisait alors figure de rivale. C'est là une grande différence, il en est d'autres. Mieux comprendre la spécificité batave implique en effet de regarder au-delà, vers les autres républiques amies.

\section{Les républiques « sœurs »}

Dépendante de la France en tant que protectorat depuis le XVII ${ }^{\mathrm{e}}$ siècle ${ }^{36}$, Genève fut la première à reconnaître la République française et à effectuer sa propre révolution : une révolution très démocratique qui eut lieu en décembre 1792, mais qui s'avéra très violente - et qui se fit indépendamment de la France ${ }^{37}$. Genève connut ainsi deux tribunaux révolutionnaires successifs, mais aussi une démocratie véritable. Le peuple genevois pouvait s'exprimer sur toutes sortes de sujets, y compris sur les uniformes de la garde nationale ${ }^{38}$. De fait, la république de Genève se flattait d'avoir « renchéri sur le modèle » et renversé en trois semaines son ancien

(34) Barras et l'ambassadeur Meyer auraient crié à la trahison. Wolfe ToNE, Writings, op.cit., III, p. 186-189 ; AN, AF III, 69, lettres de vendémiaire an VI.

(35) AN, AF III-69 et 70.

(36) Il y avait une rivalité certaine entre Genève et les départements limitrophes en matière d'horlogerie, de subsistances et d'investissements, Voir AN, AF III-68, Lettres de Desportes de messidor et thermidor an IV.

(37) Éric GolaY, Quand le Peuple devint roi, op.cit.

(38) AN, AF III-68; Lettre du 4 germinal an VI. 
édifice $^{39}$. C'est que, comme la Hollande, elle avait connu plusieurs épisodes révolutionnaires, dont celui de 1782 qui avait mené à l'exil en France ou en Angleterre des Genevois éminents. Parmi eux, le banquier Étienne Clavière qui sera un des premiers à conseiller sa réunion. Le Directoire renouera avec cette idée ${ }^{40}$. Si encore Genève avait été une république harmonieuse, mais des factions acharnées y luttaient sans cesse pour le pouvoir. L'agent français Félix Desportes en attribue la faute à « une constitution vicieuse, sous un régime constamment révolutionnaire ». Les troubles succèdent aux troubles, surtout au moment des élections, tant et si bien qu'au printemps 1798, la France opte pour la réunion. Après tout, « cette république en miniature $»^{41}$ est « un État intermédiaire » entre la France et la Suisse. Ce serait donc simplifier la carte de l'Europe que de l'avaler. Le désir d'unir Genève à la France s'est donc progressivement éveillé. Il est dans la logique entamée depuis l'automne 1792, avec la réunion de la Savoie. Mais il démontre aussi un glissement dans la politique directoriale qui ne tolère plus des alliés turbulents. Contrairement à la Hollande perçue comme trop modérée ou trop apathique ${ }^{42}$, Genève semble trop populaire et trop violente, en un mot trop révolutionnaire aux yeux des Français de l'an VI. Qui plus est, elle passe pour être « un état parasite».

La Confédération helvétique ne présentait pas les mêmes avantages et désavantages que Genève. Elle est perçue à l'époque comme républicaine, mais oligarchique. Et surtout, elle est connue pour être pauvre, et divisée en de multiples « nations »- du point de vue des mœurs, us et coutumes, langues, religions et gouvernements, puisqu'y cohabitent démocratie directe, système oligarchique, et république représentative ${ }^{43}$. La Révolution française lui a fait perdre d'importantes ressources, notamment celles qui provenaient des troupes suisses employées en France ou des subsistances qui ne sont plus importées. Mais surtout, la Suisse joue un rôle stratégique essentiel. En tant que puissance neutre, possédant une longue frontière

(39) Le Moniteur, t.15, p. 152. En fait, la Constitution ne fut acceptée par le peuple que le 5 février 1794, mais la déclaration des droits date de mai 1793, et le texte constitutionnel fut rédigé durant l'été 1793. Voir Éric GOLAY, Quand le peuple devint roi, op.cit., p.138.

(40) AN, AF III-68, Rapport de Desportes de messidor an IV.

(41) AP, vol.52, p. 544. L'image de « miniature » est de Danton, dans la séance du 17 octobre 1792. AN, AF III-68, Desportes le 2 thermidor an 4. Trois motifs ont provoqué la réunion (contrainte, mais cachée) : géopolitique, financier/économique et politique.

(42) Personne ne note la contradiction entre les deux perceptions sur les Bataves : leurs querelles violentes entre factions et la légendaire apathie ou modération d'un peuple commerçant.

(43) André Holenstein, Thomas MAISSEN et Maarten PRAK (éds.), The Republican Alternative. The Netherlands and Switzerland compared, Amsterdam, AUP, 2008 ; Antoine BROUSSY, «Regards sur la Constitution helvétique : influences croisées entre la France du Directoire et les patriotes suisses », dans Pierre SERNA, Républiques soeurs, op. cit., p. 333-348. 
au sud-est de la France, elle constitue une zone tampon. Alliée de la France, elle lui permettrait d'économiser une armée tout entière ${ }^{44}$. Une autre représentation non négligeable de la Suisse, c'est que son peuple est guerrier, féru de ses droits et privilèges, et peu enclin à se laisser dominer. On ne peut le traiter comme on traitait ou traiterait les Hollandais ou les Italiens ${ }^{45}$. Dans un premier temps, la République française souhaite donc protéger la neutralité suisse, dont elle tire profit ${ }^{46}$. Mais c'est compter sans les patriotes énergiques, et notamment Frédéric-César de La Harpe et Peter Ochs, qui font tout ce qu'ils peuvent pour libérer leur patrie du joug de Berne. Vaudois d'origine, La Harpe réside à Paris depuis l'automne 1796 et, à partir de 1797, intrigue pour persuader le Directoire exécutif de créer une république une et indivisible en Suisse. Il a l'appui du Bâlois Ochs qui rédigera la Constitution helvétique. Contre eux, d'autres défendent la création de trois républiques ${ }^{47}$. Notamment des Français tels que Félix Desportes, qui veulent borner la puissance de cet allié de la France, mais aussi des suisses, avides de conserver leurs libertés cantonales et locales, par où ils se rapprochent des fédéralistes hollandais ${ }^{48}$. Un autre projet circule en effet au cours de l'année 1798 - attribué à Johann Lukas Legrand, aussitôt qualifié de "monstrueux et incohérent $»^{49}$. Le fait est qu'il prévoit la division du pays en trois républiques. À force d'arguments et d'interventions pressantes, La Harpe parvient néanmoins à convaincre le gouvernement français à adopter l'unité. Le 25 ventôse an VI au matin, le Directoire français a opté pour trois républiques ; le soir de la même journée, les directeurs parisiens décident irrévocablement de faire de la petite fédération une Helvétique une et indivisible ${ }^{50}$. La Harpe et Ochs peuvent être satisfaits. Ces revirements témoignent clairement de l'indécision de la France à l'égard des républiques sœurs et du rôle important qu'ont joué les patriotes étrangers.

(44) Voir Réflexions sommaires sur l'invasion de la Suisse, dans LA HARPE, Correspondance, op.cit., II, p. 544-46.

(45) Les Suisses n'auraient pas « la facilité des Français à saisir la nouveauté », « l'apathie des Hollandais ou la souplesse des Italiens ». Ce serait un « peuple irritable et courageux », affirme un témoin français. AN, AF III-81.

(46) Alfred RUfER, La Suisse et la Révolution française, Paris, 1973 ; Marc H. LERNER, A Laboratory of Liberty. The Transformation of Political Culture in Republican Switzerland, Leiden/Boston, 2012.

(47) Le Directoire français ici encore hésitait entre la création d'une république une et indivisible et la division en plusieurs républiques. Si l'unité l'emporte, c'est en grande partie grâce à La Harpe. La lecture de sa correspondance est extrêmement intéressante pour comprendre l'attitude de la France.

(48) Guillaume BRUNE dans AN, AF III-81, en date du 26 et du 30 ventôse an VI.

(49) AN, AF III-81, en date du 28 pluviôse an VI.

(50) Jean-François LA HARPE, op.cit., II, p. 99-103. 


\section{Les projets français sur les pays conquis}

Cette époque est aussi celle des victoires d'Italie et de la montée en puissance des militaires. De là datent de nouvelles réflexions des Français sur ce qu'il importe de faire des pays conquis. Un de ces textes, anonyme, se demande ainsi s'il est avantageux pour la France d'étendre plus loin encore ses frontières, et surtout, si tous les peuples conquis sont mûrs pour la liberté ${ }^{51}$. Et s'ils souhaitent la liberté, ces peuples veulent-ils rester indépendants ou être réunis à «la grande famille»? Alors que Belges, Suisses et Hollandais sont plus d'une fois mentionnés et admirés pour avoir lutté en faveur de la liberté, quand il s'agit de l'Italie, les Français sont sceptiques. Ils y voient des peuples fanatisés, menés par les prêtres. Le général Clarke est ainsi fort pessimiste sur la capacité des Italiens à accéder à la liberté. Ces derniers seraient sous la coupe de l'Église, et n'auraient ni assez d'énergie, ni assez d'instruction « pour pouvoir ou vouloir être éclairé sur leurs droits $»^{52}$. Clarke en conclut qu'une révolution italienne est prématurée. Jacob Blauw, ambassadeur de Hollande auprès des puissances italiennes, partage son avis. Il désapprouve la création de ces « républiques imperceptibles », ainsi qu'il les appelle. Il serait «absurde et coupable de leur sacrifier le repos, le bonheur de la France et de ses anciens alliés, [...] le peuple n'a pris aucune part à cette révolution, [...] elle est dirigée par des hommes et sur des principes dont le général Bonaparte a déjà dû réprimer l'audace $[\ldots] \gg^{53}$. Inquiet, Blauw s'interroge sur l'issue de ce qu'il appelle « la crise de l'Italie ». Et contrairement au patriote italien Matteo Galdi, qui perçoit dans les nouvelles créations la victoire irrésistible des principes nouveaux et une uniformité ou égalité bienfaisante entre des républiques amies ${ }^{54}$, Blauw persiste à incriminer le système désastreux du Directoire qui n'a « pas de plan, pas de système, pas de force, aucune vraie énergie ». Il en conclut que, depuis quatre ans, il n'y a eu « qu'un gaspillage de ressources et d'hommes », au lieu de la paix qu'au départ, la France avait sans doute voulu établir. C'est à ce plan de paix que Blauw veut s'attacher, quitte à négocier avec l'ennemi autrichien ${ }^{55}$. C'est qu'il souhaite que disparaisse « la force métaphysique de la Révolution française et avec

(51) Quelques observations sur les pays conquis, s.d. AN, AF III-185.

(52) AN, AF III-71, Lettre de Clarke de nivôse an V.

(53) NationaalArchief, La Haye, abrégée NA 2.01.08, 337.

(54) Galdi est ici proche d'hommes comme Paine et Barlow qui rêvaient encore en 1800 d'une « grande union de républiques ». Voir Philipp ZIESCHE, Cosmopolitan Patriots. Americans in Paris in the Age of Revolution, Charlottesville, Virginia University Press, 2010, p. 144-145.

(55) Dans une missive secrète du 20 novembre 1796, Blauw écrit à son ministre : « ma mission a pour but secret d'œuvrer à un rapprochement avec l'Autriche pour se libérer de l'influence arbitraire 
elle toutes les folies afférentes $»^{56}$. Mais il n'oublie pas de mentionner ce qui distingue cette période des précédentes : la soumission croissante des civils au pouvoir militaire - ici, à Bonaparte, plus précisément.

\section{Le Batave Blauw contre les Italiens Cerrachi, Martorelli et Galdi}

Depuis plus longtemps, des patriotes italiens essayaient de persuader le Directoire parisien des avantages à conquérir leur pays. Comme les Hollandais avant eux, ils valorisaient à foison les richesses qui attendaient les vainqueurs. Dans deux mémoires, le sculpteur Cerrachi ${ }^{57}$ énumère ainsi les ressources de l'État de Rome et les subsistances à tirer des autres États italiens. Et de décrire à longueur de pages les lieux stratégiques de son pays natal et les grands moyens financiers qui permettraient de relever la situation de la France. Ce réfugié politique, contraint de s'exiler, aurait souhaité se mettre à la tête des légions gauloises et marcher sur Rome. Cerrachi n'était pas le seul à célébrer l'Italie. L'auteur anonyme des Réflexions d'un bon citoyen sur les avantages de la conquête d'Italie embellit à loisir la péninsule, évoque ses « belles contrées » et en amplifie les ressources pécuniaires ${ }^{58}$. Il est relayé par un auteur italien, Martorelli, qui lui aussi se flatte que l'Italie soit « le jardin de l'Europe ». Ce dernier recense imprudemment les revenus des divers états, chiffres à l'appui - il prévoit ainsi un gain de 30 millions dans le Milanais et de 300 millions dans l'État de Naples ${ }^{59}$. Rien de tel chez le futur diplomate Matteo Galdi, qui lui aspire à repenser l'équilibre des nouvelles puissances républicaines ${ }^{60}$. Les rapports politiques entre la République française, la République batave, les États-Unis d'Amérique et l'Italie devraient ainsi être fondés sur la justice universelle et les droits naturels de l'homme. Le pacte fédératif qu'il formule serait réciproque dans tous les domaines, que ce soit les échanges ou l'assistance. Pour conclure, il demande à la France de créer une ligue de la liberté, qui permettrait aux hommes de vivre en paix et dissuaderait

de la France et se protéger de la Prusse ». Dans ce but, il intrigue donc pour imposer son plan de paix générale à l'Autriche. NA 2.01.08, 337.

(56) NA 2.01.08, 337. Nos italiques.

(57) Sur Cerrachi et la Hollande, Arthur WeSTSTEIJN (dir.), A marblerevolutionary. The Dutch Patriot Joan Derk van der Capellen and his monument, Rome, 2011. Cerrachi avait fait le voyage d'Amérique et séjournait alors en France. Dès frimaire an III, Gédéon Muzio avait remis un rapport à la Convention qui allait dans le même sens. AN, AF III-185.

(58) AN, AF III-185.

(59) Ibidem.

(60) Le général Pommereul avait quant à lui conçu l'idée d'une fédération républicaine en Italie, composée de 5 républiques. AN, AFIII-71. 
les rois de l'Europe de continuer la guerre ${ }^{61}$. Au même moment - en juillet 1797 - le mémoire sur l'état actuel de l'Italie de Blauw est plus alarmant ${ }^{62}$. L'Italie qu'il décrit est en fermentation, avec des gouvernements dans leur enfance et dont l'avenir est incertain. Dès lors, il envisage les avantages que pourrait en tirer la Hollande et aspire à lui rendre sa place dans le commerce méridional. Seule la paix permettrait de renouveler les traités autrefois conclus avec les pays italiens. Par où il renoue avec son mémoire antérieur, dans lequel il traitait des conditions nécessaires à la pacification générale.

Les projets qui s'accumulent sur les bureaux du Directoire parisien ou sur ceux de la République batave sont donc fort divers. Dans l'ensemble, et à l'exception de Galdi, tous pensent avant tout au profit à tirer pour leur patrie spécifique. Les Suisses ne demeurent pas en reste : Ochs et La Harpe font tout leur possible pour discréditer Berne et émanciper leur canton respectif du joug que ferait porter sur eux la puissance dominante. Leur vision de l'unité et de l'indivisibilité républicaine est subordonnée à cette ambition. Aussi, et tels leurs homologues bataves ou italiens, ils font miroiter aux Français les avantages d'une alliance sincère. Ce n'est donc pas le Directoire français seul qui dirige la politique étrangère, mais de nombreux individus en périphérie, pour la plupart des étrangers intéressés à convaincre la France que leur solution est la meilleure. Il faut suivre les manœuvres de ces patriotes : irlandais - Wolfe Tone et Arthur O'Connor, notamment - ; suisses - La Harpe et Ochs, entre autres ; hollandais Meyer, Blauw, Bicker, Van Staphorst, et Valckenaer - ${ }^{63}$; italiens - Cerrachi, Muzio, Martorelli, Galdi ou Buonarotti, ou bien encore les patriotes angloaméricains, tels que Barlow, Paine, ou Monroe, sans oublier tous ceux qui ont été plus discrets - pour comprendre l'incroyable imbroglio auquel étaient confrontés les républicains français ${ }^{64}$.

(61) Sur les idées de Galdi, voir Anna Maria M. RAO, « L'espace méditerranéen dans la pensée et les projets politiques des patriotes italiens. Matteo Galdi et la "république du genre humain" », dans Droits des gens et relations entre les peuples dans l'espace méditerranéen autour de la Révolution Française, Paris, 2006, p. 115-137 ; Antonino DE FRANCESCO, « Les patriotes italiens devant le modèle directorial français » dans Pierre SERNA (dir.), Républiques sœurs, op. cit., p. 267-280.

(62) NA 2.01.08, 337.

(63) Noël signale ainsi les voyages entre La Haye et Paris de Bicker qui conspirerait avec Van Staphorst pour « circonvenir la République française en faveur des fédéralistes » et il soupçonne Valckenaer d'intriguer avec les « anarchistes ». AN AF III-69 (missive de mai 1796).

(64) Les députés des deux conseils du Directoire protégeaient tel ou tel individu selon leurs convictions politiques personnelles. Jean de Bry semble avoir été proche de La Harpe et des Italiens. Boissy d'Anglas était plutôt l'interlocuteur de Bicker. Wolfe Tone avait été bien accueilli par Clarke et Hoche. Blauw et Meyer ont des contacts étroits avec Barras, et La Harpe entretient aussi de bonnes relations avec Merlin de Douai. 
Contrairement à ce qu'affirment à longueur de pages les deux diplomates américains à La Haye et à Berlin ${ }^{65}$, la politique directoriale ne visait donc toujours pas un but précis. En 1797, des négociations avec l'Angleterre sont entamées à Lille, qui auraient pu mener à la paix. Mais là, c'est Wolfe Tone qui se plaint que l'Europe tout entière ait été « révolutionnée » excepté l'Irlande ${ }^{66}$. Lui se démène auprès des généraux français pour que la France libère sa patrie des griffes du léopard britannique. Ailleurs, c'est La Harpe qui se lamente que l'Europe se républicanise, à l'exception de la Suisse. De là leur peu de compassion ou leur indifférence envers les autres républiques sœurs. Dans ce chaos d'intrigues et de passions, comment le Directoire pouvait-il se retrouver ? Quelle politique suivre dans tel ou tel pays et quelles réformes introduire ? C'est là un élément qu'il importe de prendre en considération. Les traités de paix conclus à l'époque procurent quelques enseignements supplémentaires.

\section{Les traités de paix du Directoire}

Dans sa thèse consacrée aux relations franco-bataves, Raymond Kubben revient sur le traité de La Haye du 16 mai 1795 avant de conclure que les termes n'en sont pas aussi monstrueux que l'affirment les historiens. La plupart empruntent en effet leurs arguments aux diplomates de l'époque, Blauw et Meyer, qui en étaient scandalisés ${ }^{67}$. Il semblerait surtout que Blauw n'ait pu supporter d'être exclu des négociations. En 1795, il rêvait de devenir le sauveur de sa patrie. À force de tergiversations, son rôle et celui de Meyer furent réduits à zéro. Ils furent déconsidérés et soupçonnés d'intrigues et de corruption.

Pour bien réévaluer les termes de ce traité, mieux vaut en vérité comparer avec ceux qui suivent. Car le Directoire en conclut plusieurs. En février 1798 avec la République cisalpine - lequel traité fut lui aussi jugé draconien par les Italiens - et en août de la même année avec la République helvétique. Traité contre lequel La Harpe mena une résistance aussi tenace

(65) Adams et Vans Murray pensaient que la France voulait éloigner l'Angleterre des ÉtatsUnis, et, craignaient même qu'elle veuille s'emparer du sud de leur pays. L'idée en aurait été émise par Edmond Genet, ensuite par le général Victor Collot. G.W. KYTE, «A Spy on the Western Waters : the Military Intelligence Mission of General Collot in 1796 », The Mississippi Valley Historical Review, vol. $34, \mathrm{n}^{\circ} 3,1947$, p. 427-442.

(66) Wolfe TONE, Writings, op. cit., III, 202.

(67) Raymond KuBBEN, Regeneration and Hegemony. Franco-Batavian Relations in the Revolutionary Era, Leiden \& Boston, 2011, p. 219-223. Voir aussi les lettres de Blauw et Meyer à leur gouvernement, et les missives de celui-ci. GS, I, p. 631-678 ; p. 687-691. 
que Blauw et Meyer ${ }^{68}$. Une comparaison rapide démontre que celui qui alliait la France et la Hollande est plus sévère en vérité que ceux qui sont signés par la suite. La République batave en effet doit non seulement fournir une force navale importante, partager avec la France le port de Flessingue, céder la Flandre hollandaise, Maastricht et Venlo, les biens du Stadhouder, mais encore payer une indemnité de 100 millions de florins. Dans les articles secrets, qui plus est, l'alliée batave est contrainte d'entretenir à ses frais 25000 hommes de troupe ${ }^{69}$. Le traité de commerce dont avaient rêvé les Bataves n'est pas mentionné. L'alliance est offensive et défensive. C'est-à-dire que les Bataves doivent s'engager aux côtés de la France quand celle-ci est en guerre, notamment contre l'Angleterre. En aucun cas, elle n'a le droit de revendiquer la neutralité - ou de faire la paix avec Albion ${ }^{70}$. Enfin, les troupes franco-bataves se trouvent sous le commandement d'un général français. Cette présence française en Hollande est quelque peu contradictoire avec la proclamation de son indépendance. Mais elle n'a pas eu des conséquences aussi fâcheuses qu'en Suisse ou qu'en Italie.

Trois ans plus tard, quand le Directoire conclut à Paris son alliance avec la République cisalpine, il exige certes l'entretien de 25000 hommes par cette dernière, à raison de 18 millions de livres par an, payables en mensualités, mais le solde, l'équipement, l'habillement reviennent à la France. De la république sœur sont également exigées une réquisition de troupes italiennes, à raison de 22000 hommes minimum ${ }^{71}$, la construction de places et de fortifications, la création d'équipages et d'une flottille. Mais la France lui cède l'artillerie prise sur son territoire, et surtout, conclut un traité de commerce avec des avantages réciproques, notamment la libre circulation des marchandises et des échanges exclusifs entre les deux républiques. L'Italie donc ne cède pas de territoires et ne paie pas une indemnité gigantesque comme la Hollande. Ici encore est décrété un traité

(68) Voir les textes dans Michel KÉRAUTRET, Les Grands Traités du Consulat, Paris, 2002, p. 24-33. La Harpe avait tenté d'en modifier les termes, car il refusait une alliance offensive, incompatible avec la neutralité ancestrale de la Suisse. LA HARPE, Correspondance, op.cit., II, p. 484-486 ; p. 488-89 et p. 495-99.

(69) François CROUZET estime à 13 millions et, ensuite à 10 millions de florins l'entretien annuel des troupes stationnées en Hollande, « Aspects financiers de la relation franco-batave », dans Remous révolutionnaires, op. cit., p. 53.

(70) Or, en 1799, devant l'attaque anglo-russe et les défaites de la France, la République batave essaie secrètement de retrouver sa neutralité, en faisant intervenir la Prusse. Curieusement, Kubben n'en parle pas. Voir Simon Schama, Patriots and Liberators. Revolutions in the Netherlands, 1780-1813, New York, Knopf, 1977, p. 397-399.

(71) Michel KÉRAUTRET, Les Grands traités, op. cit., p.106-113. La République cisalpine comptait environ 3,3 millions d'habitants. La somme exigée pour l'entretien des troupes sera augmentée, et malgré cette augmentation, elles manqueront de tout durant la campagne désastreuse de 1799. 
offensif et défensif, avec ordre d'expulser les émigrés qui pourraient se trouver sur le territoire allié. Il fallut pourtant bien des menaces avant que le traité ne soit accepté ${ }^{72}$. Les Italiens en trouvaient les termes trop sévères.

Envers la République helvétique, le Directoire est plus souple encore. Aucune indemnité n'est exigée, et l'entretien des troupes n'est même pas imposé à la Suisse, puisqu'il est confié à la " puissance requérante », dont les détails seraient déterminés par des conventions spéciales ${ }^{73}$. C'est la France en réalité qui constitue cette puissance. Inversement, elle paiera les troupes suisses qu'elle lèvera. Elle promet par ailleurs de protéger son alliée de l'oligarchie indigène, et des velléités belliqueuses des grandes puissances. En compensation, elle exige la libre circulation en territoire suisse sur deux voies à délimiter. L'une menant en Allemagne, l'autre en Italie. La libre circulation est accordée aux citoyens suisses qui désirent se rendre en France et qui y jouiront des mêmes droits que les citoyens français, ce qui suggère une réciprocité certaine. La reconfiguration des frontières tient également à cœur aux Français, qui viennent d'annexer la république de Genève. Depuis Bâle jusqu'à Genève donc, des rétrocessions et échanges sont prévus en vue d'une « rectification plus parfaite» des limites des deux républiques. Des articles secrets prévoient ainsi de réunir le Frickhal à la république helvétique, afin d'arrondir « ses frontières à son avantage », et la France promet de retirer ses troupes du territoire suisse dès que possible et d'entretenir elle-même celles qui resteront sur place.

Ce texte d'août 1798 n'était pas l'original conçu par le Directoire. Un premier projet encore plus avantageux, inspiré sans aucun doute par La Harpe, avait été rédigé en juillet 1798 et porté à la connaissance de l'agent français en Suisse, le fameux Rapinat. Il prévoit de garantir l'intégrité du territoire suisse, le maintien de la neutralité, l'armement à titre gratuit des troupes suisses, et leur paiement par la France. Enfin, il stipule que les ministres helvétiques assistent et délibèrent « aux conférences où les négociations sont commencées et suivies jusqu'à la conclusion de la paix ». Sur tous ces points, Rapinat trouve à redire. Tout d'abord, l'annexion de Genève entraîne avec elle une reconfiguration des frontières, ce qui fait que celles de la Suisse devront être modifiées, d'autant que vont être ouvertes

(72) Raymond GuYOT, Le Directoire et la paix en Europe, op. cit., p. 796-806.

(73) Michel KÉRAUTRET, Les Grands Traités, op.cit., p.114-121. Des contributions de guerre furent pourtant levées dans la plupart des cantons. Au total 14 millions de francs. Voir Jacques GODECHOT, Les commissaires aux armées, op.cit., II, p. 58-63, p. 131-147 ; Raymond GUYOT, Le Directoire et la paix, op. cit., p. 740-767. 
des voies de passage vers l'Italie. Sur la neutralité, le diplomate est tout aussi critique :

« La Suisse doit être l'amie de la France et sa fidèle alliée, et vice versa », ce qui implique une alliance offensive et défensive ${ }^{74}$. Rapinat ne comprend pas non plus l'urgence d'armer les Suisses, à un moment où les troubles sont légion dans les campagnes. Mieux vaudrait y cantonner 12000 Français. Quant au paiement prévu pour les soldats suisses, il serait franchement exorbitant - un million pour chaque mille hommes. Ce serait « revenir à l'Ancien Régime et retomber dans ses habitudes ».

Il propose donc de les solder comme les troupes françaises. Sur les articles secrets, il est tout aussi perplexe, notamment sur la présence des Suisses lors des négociations : «il paraîtrait singulier de forcer le gouvernement français à appeler les ministres helvétiques aux délibérations qui précéderaient les traités à conclure entre la France et les ennemis de l'Helvétie, puisque ces ministres seraient témoins et connaîtraient les articles secrets qu'il serait peut-être important à la France de laisser ignorer aux Suisses $»^{75}$. Les critiques ont été entendues par le Directoire. Le traité définitif a supprimé ou modifié ces articles et les a adaptés aux suggestions du diplomate. Le dernier point sur les négociations n'est plus mentionné. Le paiement des troupes suisses se fera sur le mode français. Il n'est plus question de neutralité, mais, comme en Hollande et en Italie, d'alliance offensive et défensive.

Ce que révèlent ces divers traités, ce sont les rapports de force entre alliés, mais aussi les perceptions que se font les Français du pays qui vient d'être "libéré ». En Hollande, le traité sanctionne la fin de la guerre et souhaite « en réparer les maux par une juste distribution de dédommagements et d'avantages réciproques ». L'alliance se fait contre l'Angleterre et le stadhouder ${ }^{76}$. Depuis les tout débuts de la Révolution française, la France est persuadée que la Hollande est un vaste entrepôt, ruisselant d'or et de marchandises, et compte bien en profiter. Inversement, la Hollande qui est durement imposée dès les débuts, a la chance de ne pas avoir été sans cesse spoliée, comme il en est allé en Italie et en Suisse, où les généraux et les agents divers se servaient plus que généreusement. La

(74) Les commentaires de Rapinat sont publiés dans LA HARPE, Correspondance, op.cit., II, p. 484-486 et p. 495-499.

(75) Jean-François LA HARPE, Correspondance, op. cit., II, p. 547-555. Ceci explique pourquoi les alliés de la République française ne sont pas invités aux négociations préliminaires, et répond à la question que se posait KUBBEN, Regeneration and Hegemony, op. cit., p. 689-695.

(76) Michel KÉRAUTRET, Les Grands Traités, op.cit., I, p. 25. En Suisse, la guerre est imputée aux oligarchies (Ibidem., p.115), et en Italie, la cause n'est même pas mentionnée (Ibid., p.107). 
Suisse, cependant, passait pour être pauvre. Le traité d'alliance en tient compte. Elle n'en est pas moins maltraitée par les généraux français sur place, qui ne cessent d'exiger des sommes importantes, à titre d'emprunts. C'est ainsi que Soult demande 300000 francs à la ville de Saint-Gall. Il succède à Ménard qui a levé 700000 francs et à Brune, qui part en Italie avec 400000 francs pour ses dépenses secrètes particulières. Les plaintes s'accumulent ${ }^{77}$. Treize mois après le traité d'alliance, la Suisse se plaint de n'avoir pu remplir ses engagements à cause des contributions, des réquisitions et des frais occasionnés par le passage des troupes. Mais c'est en Italie que, progressivement, se met en place un terrible système de spoliation et de dilapidation. Tout ce qui est diamant, argenterie et bijoux par exemple est curieusement omis des états de comptes. En conséquence, ils se volatilisent. Dans un rapport officiel de 1799, le ministre de la Guerre, Bernadotte calcule que l'Italie a dû fournir en un an plus de 62 millions de francs et la Suisse pas moins de 14. Et il conclut tristement : « et tout a disparu $\gg^{78}$.

Ce gâchis n'a pas eu lieu en Hollande, où les gouvernements successifs ont su freiner les prétentions des généraux français ${ }^{79}$. Il y a bien eu quelques gaspillages et corruptions durant le régime radical de janvier-juin 1798. Mais les sommes ne furent en rien comparables aux dilapidations commises en Suisse, et avant tout, en Italie. De ce point de vue, et comme le constate Kubben, l'alliance franco-batave aurait pu être envisagée plus positivement que ne le font d'ordinaire les historiens. D'autant que seule (ou presque) parmi les républiques alliées, la République batave a eu la liberté de se donner une constitution. Mais ce sont justement les problèmes liés à cette constitution qui inciteront la France à mener une politique beaucoup plus dirigiste dans les autres républiques soeurs.

\section{Des constitutions libres ou sur mesure ?}

Une fois le traité d'alliance signé en mai 1795, la République batave était donc libre de se donner une constitution. Deux ans plus tard, le peuple hollandais rejette le texte en question. Plusieurs mois s'écoulent, et les

(77) Sur la levée de contributions de guerre, voir les détails par Rapinat, dans AN, AF III-81. Ces contributions ne sont pas mentionnées dans les traités. Sur les plaintes des Suisses, LA HARPE, Correspondance, op.cit., II, 523.

(78) AN, AF III-81, Rapport du ministre de la Guerre du 28 messidor an VII.

(79) En 1799, William VANS MURRAY signale pourtant la remise de deux millions de florins à Fouché. Letters of William Vans Murray, Tenth Annual Report of the American Historical Association, 1912, p. 578. Sur les dilapidations du gouvernement radical, voir Authentique Bijlagentot den gebeurtenissen van den 12 Juni, La Haye, 1799. 
Bataves ne parviennent toujours pas à s'accorder. Entre-temps, le directoire français fulminait que son alliée n'ait pas d'existence politique et ne puisse mettre en œuvre une politique énergique. Ce qui passait pour inertie ou indifférence est cause de l'écoute favorable que prête peu à peu le Directoire exécutif aux radicaux bataves. Les Français ne sont pas déçus : en quelques semaines, le projet de constitution est achevé et accepté. Or ce projet résulte des travaux de la commission batave de constitution ${ }^{80}$. Contrairement à ce qui se passe dans le même temps à Paris, où Merlin de Douai et Daunou corrigent le projet de Peter Ochs et repensent les constitutions italiennes, les observations françaises sur le projet batave n'ont pas été ou très peu prises en considération. Personne ne s'en est plaint. L'essentiel était que la Hollande ait une constitution.

En vérité, Genève fut la première république alliée à concevoir sa propre constitution, précédée d'une déclaration des droits de l'homme, étonnamment moderne ${ }^{81}$. L'introduction en fut assez facile, mais la démocratie qu'elle introduisait s'avéra peu viable. Suivirent en 1796-1797 les républiques italiennes, dont celle de Gênes - la république ligurienne, qui rédigea elle-même son texte de lois - et la Cisalpine, qui se vit imposer un texte par Bonaparte. Mais, bientôt il s'avéra que ce texte-ci n'était pas satisfaisant ${ }^{82}$. Un des partisans de l'Italie républicaine, le secrétaire de légation Bignon, note ainsi que la constitution de la Cisalpine donnée par Bonaparte avait été « rédigée à la hâte, au milieu des camps ${ }^{83}$. Il fallait le corriger. À partir de là, la République française intervint plusieurs fois en matière de constitution dans les États italiens. Ainsi Rome sert-elle de laboratoire à Daunou qui conçoit un texte plus ou moins adapté au contexte $^{84}$. Le cas de la République helvétique est quant à lui spécifique, et on l'a trop peu souligné. Car c'est La Harpe qui insistait pour proposer à la Suisse une constitution « toute prête » afin d' « accorder tant d'intérêts

(80) A ce sujet, Annie JouRdan, La Révolution batave entre la France et l'Amérique, Rennes, PUR, 2008.

(81) La déclaration genevoise proclamait la garantie sociale et le droit de réunion. Voir $L e$ Moniteur, t. 16, p. 557, Lettre du 15 mai 1793. Voir le texte dans Éric GoLAY, Quand le peuple devint roi, op.cit., p. 638-642.

(82) Claude Joseph Trouvé, Sur la République cisalpine, Paris, an VII, p.15. Trouvé explique ce qui fut modifié : les cercles populaires furent fermés, les journaux placés sous contrôle du gouvernement, les départements fixés à 11 et les députés réduits à 120 .

(83) Il y avait à l'origine 21 départements et 224 représentants du peuple, ce qui faisait beaucoup pour une république de trois millions d'habitants. Voir les critiques de Louis Pierre Édouard BIGNON, Du système suivi par le Directoire exécutif relativement à la République cisalpine, Paris, an VII, p.12-16.

(84) Voir le texte imprimé et les corrections dans AN, AF III-78, Constitution de la République française de 1795 adaptée à la République romaine. 
divers $»^{85}$. Début décembre 1797, il avouait déjà vouloir arrêter les bases de cette constitution avec le Directoire parisien. Il y eut donc coopération d'un côté comme de l'autre. Mais le texte final est bien celui de Ochs. Merlin de Douai l'a certes corrigé sur des points de détail, et surtout pour supprimer ce qui rappelait trop l'Ancien Régime ou pour «moderniser » la formulation. Une analyse du texte et des corrections en témoigne. Merlin n'a modifié ni les principes fondamentaux posés en préambule à la Constitution helvétique, ni l'organisation des pouvoirs conçus par Ochs ${ }^{86}$.

Il y a donc des différences non négligeables entre les républiques sœurs. Mais la République batave ressort de cette vue d'ensemble comme étant plus autonome que celles qui sont venues plus $\operatorname{tard}^{87}$. Il s'avère également que les interventions de la France avaient moins pour but d'imposer ses propres principes que d'accélérer l'organisation des républiques alliées et d'en rationaliser le fonctionnement. À cet égard, la République batave était le modèle à éviter. C'est également ce que suggèrent les propos de La Harpe ${ }^{88}$. Lui comprend qu'il faut tout d'abord un texte de lois, sous peine de subir des ingérences françaises ou de prolonger à n'en plus finir la révolution. L'exemple batave hante son esprit - et sans doute aussi les tribulations de Genève. La France a donc fini par se mêler des affaires de ses alliés, parce qu'elle a expérimenté combien étaient laborieux ou conflictuels les travaux des nations en révolution. Or elle avait besoin de leur soutien dans une guerre qui n'en finissait pas.

Une étude de la « grande famille » républicaine confirme avant tout que les révolutions et leurs constitutions ne rallient pas l'ensemble des citoyens, fussent-ils patriotes. Ils se déchirent sur les principes, sur le mode d'organisation, la fiscalité, la souveraineté, l'amalgame ou l'unité. Dans les républiques sœurs, les textes et nouveautés révolutionnaires étaient loin de faire l'unanimité. Des partis s'affrontaient dans une lutte qui ressemblait à une guerre civile, ce qui fut donc le cas à Genève. Dans la

(85) Jean-François LA HARPE, Correspondance, op. cit., I, p. 402-403 ; II, p. 383-85 et p. 422. La Harpe ne voulait pas perdre de temps afin de ne pas mettre l'indépendance de la Suisse en péril et d'éviter la lutte des factions. Il pensait que la Constitution pouvait être progressivement améliorée.

(86) Notons que Merlin supprime tout ce qui pourrait suggérer le provisoire. Il modernise la définition de la citoyenneté - il n'y a plus de citoyens nés de citoyens, mais est citoyen tout homme âgé de 20 ans qui prête serment et se fait inscrire sur le registre civil. Voir le texte original avec les corrections dans AN, AF III-185. Plus de détails dans Hervé LEUWERS, Un juriste en politique, op.cit., p. 231-232 et Antoine BROUSSY, « Regards sur la Constitution helvétique » dans Pierre SERNA, Républiques sœurs, op. cit., p. 333-348.

(87) Sur la constitution très originale de Naples, voir Anna-Maria RAO, La Repubblica Napoletana del 1799, Naples, 1999.

(88) LA HARPE, Correspondance, op.cit., I, 364 et p.372. Wolfe TonE est tout aussi négatif sur le déroulement de la révolution batave, Writings, op. cit., III, p. 186-187 et p. 193-194. 
République batave aussi, la guerre civile menaçait et a été évitée grâce aux interventions des armées françaises ${ }^{89}$. En Suisse, ce sont elles encore qui ont affronté les cantons hostiles. Les correspondants helvétiques de La Harpe l'avouent explicitement quand ils constatent que la présence française leur a épargné une " guerre civile $»^{90}$. Contrairement à Adams et Vans Murray, qui condamnaient la «terrible République » pour son interventionnisme croissant, les patriotes étrangers se félicitaient que la présence française ait évité l'effusion de sang. La même chose vaut en somme en Italie où la retraite des troupes françaises de 1799 permit aux contre-révolutionnaires de prendre leur revanche. Les patriotes qui étaient restés sur place apprirent à leurs dépens ce qu'il en était. À Naples, les contre-révolutionnaires ont même violé la promesse d'amnistie qu'ils avaient faite aux révolutionnaires et ont massacré tous ceux - hommes ou femmes - qui leur avaient fait confiance $^{91}$. Le cas de Genève est également éloquent. Jusqu'à sa réunion à la France d'avril 1798, s'y étaient succédées des scènes de violence, des condamnations à mort, des bannissements. Il s'avérait ainsi que les révolutions divisaient les populations en factions féroces et que la guerre civile était inévitable. En Hollande même, la répression sévère qui s'abattit en janvier et en juin-juillet 1798 sur les adversaires politiques témoignait d'une soif de vengeance inattendue - que revendiquaient plus fort encore les radicaux populaires ${ }^{92}$. La France, qui avait appris sa leçon, fit tout ce qui était en son pouvoir pour éviter à ses alliés de tomber dans le même piège. Tant qu'elle eut son mot à dire, vengeance et terreur ne parvinrent pas à s'imposer dans les républiques sœurs ${ }^{93}$. Ce qui compensait quelque peu les abus de pouvoir, les contributions forcées, les spoliations, et les problèmes dus à la présence de troupes. Au terme de cette étude, force est donc de constater que la République batave ne fut pas seulement une exception dans la constellation des républiques sœurs. Bien qu'elle ait moins souffert que ses « sœurs » et que son autonomie fût plus grande, certains traits - qu'on peut appeler révolutionnaires - leur sont à toutes communs. Ces affinités ne furent pourtant pas une raison pour les autres républiques de la prendre pour exemple. Bien au contraire. En raison des problèmes qui se posèrent

(89) Les agents français évoquent la crainte d'une guerre civile, mais aussi ADAMS, I, p. 317320 et p. 331

(90) LA HARPE, Correspondance, op.cit., I, 417-420. Lettre de Secretan.

(91) Voir Anna-Maria RAO, La Repubblica Napoletana, op.cit., p. 58-62.

(92) À ce sujet, JouRdAn, La Révolution batave, op. cit., p. 397-434.

(93) La politique d'exception menée par les radicaux entre janvier et juin 1798 fut traitée de « terroriste » par leurs adversaires. VoirVerantwoording van Pieter Vreede aan de Bataafsche Natie, Leyde, 1798. 
pour introduire une constitution, œuvrer à la réconciliation nationale et manifester sa puissance guerrière, elle fut un modèle à éviter. Il fallut la victoire contre les Anglo-Russes de l'automne 1799 pour qu'elle retrouve un certain éclat. Seule parmi les républiques sœurs, elle avait su tenir tête à l'invasion ennemie ${ }^{94}$.

Annie JOURDAN

Université d'Amsterdam

Études européennes

Spuistraat 134

NL -1012 VB-Amsterdam a.r.m.jourdan@uva.nl

(94) On ignorait alors que le gouvernement avait envoyé un agent en Prusse en vue d'obtenir la neutralité. Quant à la victoire, le général Brune y était pour beaucoup. Voir Simon ScHAMA, Patriots and Liberators, op.cit., p. 397-399. 